\title{
Construction of the Dirichlet to Neumann Boundary Operator for Triangles and Applications in the Analysis of Polygonal Conductors
}

\author{
Thomas Demeester and Daniël De Zutter, Fellow, IEEE
}

\begin{abstract}
This paper introduces a fast and accurate method to investigate the broadband inductive and resistive behavior of conductors with a non-rectangular cross-section. The presented Iterative Combined Waveguide Modes (ICWM) algorithm leads to an expansion of the longitudinal electric field inside a triangle, using a combination of parallel-plate waveguide modes in three directions, each perpendicular to one of the triangle sides. This expansion is used to calculate the triangle's Dirichlet to Neumann boundary operator. Subsequently, any polygonal conductor can be modeled as a combination of triangles. The method is especially useful to investigate current crowding effects near sharp conductor corners. In a number of numerical examples, the accuracy of the ICWM algorithm is investigated, and the method is applied to some polygonal conductor configurations.
\end{abstract}

Index Terms-Dirichlet to Neumann operator, iterative combined waveguide modes algorithm, triangle, polygonal conductor, skin effect, quasi-TM approximations.

\section{INTRODUCTION}

$\mathbf{T}$ O adequately address broadband signal integrity for board and package level interconnections, fully fledged RLGC transmission models are required. For the highest clock rates this is now also the case for on-chip interconnections as argued in [1]. The influence of the finite conductivity of the conductors and the associated frequency dependent skin effect losses and internal inductance (the so-called current crowding phenomenon) has received considerable attention in literature, see e.g. [2] and [3] and the many references in these papers. In [4] a single lossy line in the presence of a semiconducting substrate is analysed in the quasi-TM limit. The conductor losses can even become dominant for narrow strip configurations [5]. In [6] this quasi-TM analysis is extended to multiconductor lines in the presence of a semiconducting substrate.

To this end we introduced the Dirichlet to Neumann (DtN) operator [7] to capture the current crowding phenomenon inside a good conductor. The frequency ranges from DC to tens of $\mathrm{GHz}$, at which point the skin-effect is fully developed and can be described by the familiar scalar surface impedance $Z_{s}$

$$
Z_{s}=\frac{1+j}{\sigma \delta}
$$

with $\sigma$ the conductivity, and $\delta$ the skin depth. The DtN operator is used to obtain a surface admittance relationship $\mathcal{Y}\left(\boldsymbol{r}, \boldsymbol{r}^{\prime}\right)$

The authors are with the Department of Information Technology, Ghent University, Sint-Pietersnieuwstraat 41, B-9000 Gent, Belgium. Tel: +32 9264 33 27, Fax: +329264 35 93. E-mail: thomas.demeester@intec.ugent.be, daniel.dezutter@ugent.be between the longitudinal electric field $e_{z}\left(\boldsymbol{r}^{\prime}\right)$ and the differential surface current $j_{s}(\boldsymbol{r})$, with $\boldsymbol{r}^{\prime}$ and $\boldsymbol{r}$ on the circumference of the conductor's cross-section. To determine the per unit length inductance and resistance of a particular transmission line configuration, it now suffices to replace the conductors by their equivalent differential surface currents placed in the background medium. These differential, or so-called 'equivalent' surface currents are determined such, that they exactly give rise to the original fields outside the conductor when this conductor is made transparent by replacing its material properties by those of the background medium. Combining this with an integral equation solution for the fields generated by these currents, directly leads to the desired $L$ and $R$ matrices. In the quasi-TM approximation this approach can be extended to the determination of the capacitance and conductance matrices $\mathrm{C}$ and $\mathrm{G}$ by again invoking the DtN operator but now to obtain a relationship between the potential $\phi$ on the circumference of each dielectric and semiconductor, and its normal derivative $\partial \phi / \partial n$. Invoking an integral equation for the potential as a function of the equivalent surface charges in combination with this relationship between $\phi$ and $\partial \phi / \partial n$, and by exciting the conductors' boundaries with a constant potential, allows for a determination of $C$ and $G$ as demonstrated in [6] for coupled lines in the presence of semiconducting media.

Although the DtN operator theory can in principle be applied to conductors with an arbitrary cross-section, its practical application remained restricted to a rectangular cross-section. This is due to the fact that the analytical determination of the DtN operator was based on its expansion in terms of the Dirichlet eigenfunctions. These functions are only known for the rectangle and the circle and because at least a few thousands of them are needed for a correct broadband analysis, their numerical determination for other shapes is excluded. However, more complex conductor shapes, provided they are formed by combining rectangles, can be handled as demonstrated in [8] and [9], e.g., to examine the effect of layered on-chip conductors.

Effects such as underetching or electrolytical growth in the integrated circuit manufacturing process, lead to conductors that are trapezoidal rather than rectangular. In [10], such lines are investigated using a combination of the Finite Element Method (FEM) and the Method of Lines (MoL). To investigate trapezoidal conductors, or more generally, the influence of sharp or obtuse conductor corners on the current crowding, by means of the fast boundary integral equation method presented in [6], the knowledge of the DtN operator for a 
triangular cross-section is of paramount importance. Combining triangular cross-sections with rectangular ones, as in [9] for rectangular cross-sections, then allows one to approximate almost any cross-sectional shape.

In section II and III the DtN operator for the triangle is determined by a new method not depending on the Dirichlet eigenfunctions. As argued and demonstrated in [6] and [7], for piecewise homogeneous media, the use of the DtN operator allows to reformulate the complete problem in terms of a set of coupled boundary integral equations only requiring the discretization of the unknowns on the boundaries of the different subdomains. The use of the proper Green's functions for each subdomain, leads to the additional advantage that the skin-effect can be captured in a very accurate way.

First, the $e_{z}$ field on the circumference of the triangle is discretized, e.g., by using piecewise constant or linear basis functions. Inside the triangle $e_{z}$ satisfies the diffusion equation. Next, we choose to expand $e_{z}$ inside the triangle in terms of three sets of parallel-plate waveguide modes, each set with one of the triangle's sides as the waveguide's height. In theory using only a single complete set of parallel-plate waveguide modes would suffice, but the extra waveguide modes are introduced to avoid numerical inaccuracies as will be carefully substantiated at the end of Section II-A. This in turn leads to a numerically very stable determination of the normal derivative of $e_{z}$ on the circumference, as a function of its original discretized representation.

In section IV, some numerical examples demonstrate the accuracy of the method and the convergence properties of the iterative process to determine the DtN operator. Furthermore, the resistance of a single conductor composed of triangles is determined for different conductor shapes. For a rectangle, results are compared with data available in literature. Next, the resistive and inductive properties of a multiconductor line with trapezoidal conductors are investigated and compared to the rectangular conductor case. Finally, a coplanar waveguide above a non-planar substrate is simulated and its characteristic impedance is compared with reference data.

\section{Determination of The DtN Operator IN A TRIANGLE}

As introduced in [7] and further elaborated in [6], the required relationship between $e_{z}$ on a triangle's boundary $c$ and the differential surface current $j_{s}$ is given by

$$
\begin{aligned}
j_{s}(\boldsymbol{r}) & =\frac{1}{j \omega \mu_{0}}\left(\frac{\partial e_{z}(\boldsymbol{r})}{\partial n}-\frac{\partial e_{z, 0}(\boldsymbol{r})}{\partial n}\right), \quad \boldsymbol{r} \in c \\
& =\frac{1}{j \omega \mu_{0}} \oint_{c}\left(\mathcal{D}\left(\boldsymbol{r}, \boldsymbol{r}^{\prime}\right)-\mathcal{D}_{0}\left(\boldsymbol{r}, \boldsymbol{r}^{\prime}\right)\right) e_{z}\left(\boldsymbol{r}^{\prime}\right) d c\left(\boldsymbol{r}^{\prime}\right)
\end{aligned}
$$

with $\left(\mathcal{D}-\mathcal{D}_{0}\right)$ the differential $\mathrm{DtN}$ operator. The actual electric field $e_{z}$ and the fictitious field $e_{z, 0}$ have the same boundary value on $c$, but inside triangle $T, e_{z}$ satisfies the diffusion equation, whereas $e_{z, 0}$ satisfies Laplace's equation

$$
\begin{aligned}
\nabla_{t}^{2} e_{z}(\boldsymbol{r}) & =j \omega \mu_{0} \sigma e_{z}(\boldsymbol{r}), \quad \boldsymbol{r} \in T \\
\nabla_{t}^{2} e_{z, 0}(\boldsymbol{r}) & =0, \quad \boldsymbol{r} \in T
\end{aligned}
$$

as dictated by the quasi-TM approximations [6]. For a rectangular area, (3) was discretized by means of the Dirichlet expansion of $\left(e_{z}-e_{z, 0}\right)$, which is zero on the boundary $c$.

For the complex capacitance problem $\mathrm{C}+\mathrm{G} / j \omega$, the required relationship between the equivalent surface charge $\rho_{s}$ and the electric potential $\phi_{c}$ on the boundary of dielectrics and semiconductors is given by

$$
\begin{aligned}
\rho_{s}(\boldsymbol{r}) & =\left(\epsilon-\epsilon_{0}+\sigma / j \omega\right) \frac{\partial \phi(\boldsymbol{r})}{\partial n}, \quad \boldsymbol{r} \in c \\
& =\left(\epsilon-\epsilon_{0}+\sigma / j \omega\right) \oint_{c} \mathcal{D}\left(\boldsymbol{r}, \boldsymbol{r}^{\prime}\right) \phi\left(\boldsymbol{r}^{\prime}\right) d c\left(\boldsymbol{r}^{\prime}\right)
\end{aligned}
$$

in which $\phi$ satisfies the diffusion equation in the semiconductors, and Laplace's equation in the dielectrics. The discretization of (7) requires the determination of the non-differential DtN operator $\mathcal{D}$. Because a Dirichlet expansion cannot be used to represent a non-zero boundary function, an alternative expansion was used to discretize (7) on a rectangle [11], based on the superposition of the modal fields that exist in two perpendicular parallel-plate waveguides. A similar approach will be used here, but the contributions from the three parallelplate waveguides, each perpendicular to one of the sides of the triangle, will interfere with one another, which was not the case for the rectangle.

The algorithm introduced in this paper will lead to the DtN matrix $D$, the discretized form of the non-differential operator $\mathcal{D}$, defined for triangle $T$ with boundary $c$ by

$$
\frac{\partial \psi(\boldsymbol{r})}{\partial n}=\oint_{c} \mathcal{D}\left(\boldsymbol{r}, \boldsymbol{r}^{\prime}\right) \psi\left(\boldsymbol{r}^{\prime}\right) d c\left(\boldsymbol{r}^{\prime}\right), \quad \boldsymbol{r} \in c
$$

for $\psi$ satisfying

$$
\nabla_{t}^{2} \psi(\boldsymbol{r})=-k^{2} \psi(\boldsymbol{r}), \quad \boldsymbol{r} \in T .
$$

\section{A. Geometry of the Problem and Expansion of $e_{z}$}

Consider triangle $T$, shown in Fig. 1, defined by its corner points $p_{1}(0,0), p_{2}\left(0, x_{0}\right)$ and $p_{3}\left(x_{1}, y_{0}\right)$. Along the sides $c_{1}$, $c_{2}$ and $c_{3}$, we will use the normalized coordinate $s$ ranging from 0 to 1 in counter-clockwise direction along boundary $\mathrm{c}$ of triangle $T$, such that

$$
\begin{aligned}
& c_{1} \leftrightarrow \quad\left\{x=s x_{0}, y=0\right\} \\
& c_{2} \leftrightarrow\left\{x=x_{0}+s\left(x_{1}-x_{0}\right), y=s y_{0}\right\} \\
& c_{3} \leftrightarrow \quad\left\{x=(1-s) x_{1}, y=(1-s) y_{0}\right\}
\end{aligned}
$$

The outward pointing normal unit vectors on each side are needed in the sequel as well. They are given by

$\mathbf{u}_{1}=[0,-1], \mathbf{u}_{2}=\left[\frac{y_{0}}{l_{2}}, \frac{x_{0}-x_{1}}{l_{2}}\right], \mathbf{u}_{3}=\left[-\frac{y_{0}}{l_{3}}, \frac{x_{1}}{l_{3}}\right]$

with $l_{1}, l_{2}$ and $l_{3}$ the lengths of the respective sides.

The function $\psi(x, y)$ inside $T$ is split up into three subfunctions

$$
\psi(x, y)=\psi^{(1)}(x, y)+\psi^{(2)}(x, y)+\psi^{(3)}(x, y)
$$




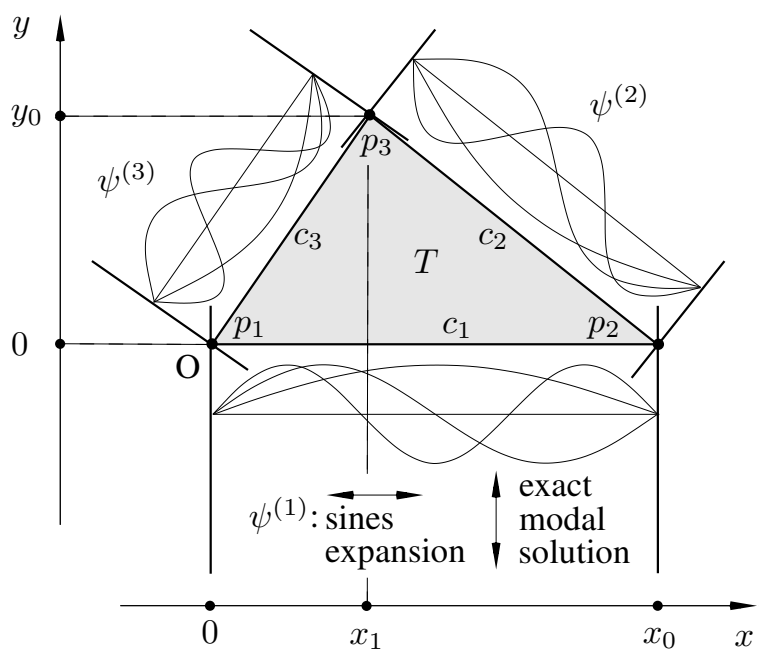

Fig. 1. Triangle $T$, with corners $\left\{p_{i}\right\}$ and sides $\left\{c_{i}\right\}(i=1,2,3)$, placed in a cartesian coordinate system with origin $\mathrm{O}$ and axes $(x, y)$, and with a schematic indication of the way $\psi$ on $T$ is split up into $\psi^{(1)}, \psi^{(2)}$, and $\psi^{(3)}$, according to parallel-plate waveguide modes in three directions.

with

$$
\begin{aligned}
& \psi^{(1)}(x, y)=\sum_{n=1}^{N_{1}} A_{c_{1}, n} f_{c_{1}, n}(x, y) \\
& \psi^{(2)}(x, y)=\sum_{n=1}^{N_{2}} A_{c_{2}, n} f_{c_{2}, n}(x, y) \\
& \psi^{(3)}(x, y)=\sum_{n=1}^{N_{3}} A_{c_{3}, n} f_{c_{3}, n}(x, y)
\end{aligned}
$$

The functions $f_{c_{1}, n}(x, y)$ are given by

$$
f_{c_{1}, n}(x, y)=\left(e^{j \beta_{n} y}-e^{-j \beta_{n}\left(y-2 y_{0}\right)}\right) \sin \frac{n \pi x}{x_{0}}
$$

with $\beta_{n}^{2}=k^{2}-\left(n \pi / x_{0}\right)^{2}$ and its square $\operatorname{root} \beta_{n}$ chosen such, that $\operatorname{Re}\left(j \beta_{n}\right)<0$. The upper limit $N_{1}$ in (15) is the number of sine functions used to expand the $x$-dependence of $\psi^{(1)}$.

The function $\psi^{(1)}$ can, with (15) and (18), be seen as an expansion of a longitudinal electric field into the eigenmodes of a parallel-plate waveguide, filled with the medium with wave number $k$ and directed vertically with the plates through corners $p_{1}$ and $p_{2}$ of triangle $T$. The $y$-dependence in (18) is the exact solution to (9) for each term in the sine expansion along $x$, and is chosen such, that its contribution at $y=y_{0}$ (and hence at $p_{3}$ ) becomes zero.

The functions $f_{c_{2}, n}(x, y)$ and $f_{c_{3}, n}(x, y)$ can be written in a similar way, but it is unnecessary to explicitly write them down in the same coordinate system used for $f_{c_{1}, n}(x, y)$. Instead, a different set of axes is associated with each side $c_{i}$ of the triangle. It has corner $p_{i}$ as its origin, and side $c_{i}$ as its $x$-axis. The same triangle in three different orientations and for each of these coordinate systems, is shown in Fig. 2.

By this judicious choice of the axes, we only need (18) to express the contributions of the parallel-plate waveguides associated wich sides $c_{2}$ and $c_{3}$, in the sense that

$$
\begin{aligned}
& f_{c_{2}, n}(x, y)=f_{c_{1}, n}^{\prime}\left(x^{\prime}, y^{\prime}\right) \\
& f_{c_{3}, n}(x, y)=f_{c_{1}, n}^{\prime \prime}\left(x^{\prime \prime}, y^{\prime \prime}\right)
\end{aligned}
$$

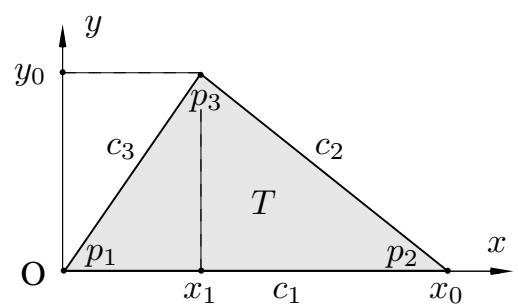

(a)

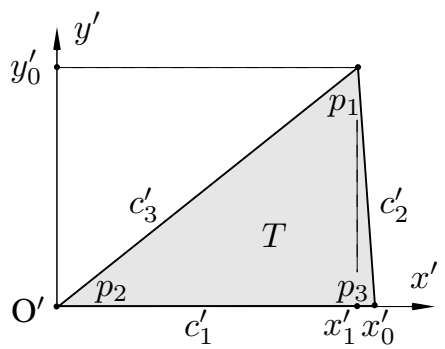

(b)

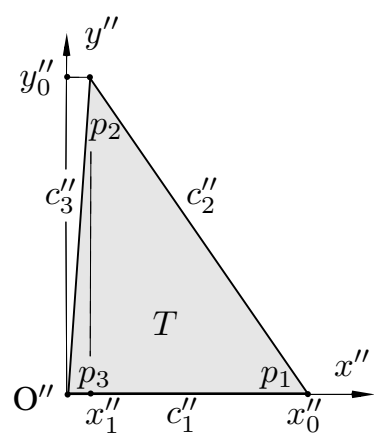

(c)
Fig. 2. Three different axes sets, each associated with a different orientation of triangle $T$. (a) unprimed, with $c_{1}$ underneath, (b) primed, with $c_{2}$ underneath $\left(c_{1}^{\prime}=c_{2}, c_{2}^{\prime}=c_{3}, c_{3}^{\prime}=c_{1}\right)$, (c) double primed, with $c_{3}$ underneath $\left(c_{1}^{\prime \prime}=c_{3}, c_{2}^{\prime \prime}=c_{1}, c_{3}^{\prime \prime}=c_{2}\right)$.

This means that, e.g., for $f_{c_{2}, n}(x, y)$, the same form as (18) is used, but with $x, y, x_{0}, x_{1}, y_{0}$ replaced by $x^{\prime}, y^{\prime}, x_{0}^{\prime}, x_{1}^{\prime}$, $y_{0}^{\prime}$, and with $N_{1}^{\prime}=N_{2}$ terms in the expansion of $\psi^{(2)}$.

The sine expansion $\left\{\sin \left(n \pi x / x_{0}\right)\right\}$ in (18) for $\psi^{(1)}$ forms (theoretically, for $N_{1} \rightarrow \infty$ ) a complete set on $c_{1}$, except for the corner points where all sine functions are zero. This problem and some related issues will be addressed in Section III. An analogous argumentation is valid for $\psi^{(2)}$ and $\psi^{(3)}$. Due to the specific form of (15) and (18), with a single multiplicative degree of freedom for each basis function $f_{c_{1}, n}$ (its coefficient $\left.A_{c_{1}, n}\right), \psi^{(1)}$ is fully determined over the complete triangle, once its boundary value on $c_{1}$ is fixed, and so are $\psi^{(2)}$ for side $c_{2}$ and $\psi^{(3)}$ for $c_{3}$. It would, alternatively, be possible to determine the coefficients $\tilde{A}_{c_{1}, n}$ and $\tilde{B}_{c_{1}, n}$ of only one complete set of waveguide modes, written as

$$
\tilde{\psi}(x, y)=\sum_{n=1}^{N_{1}}\left(\tilde{A}_{c_{1}, n} e^{j \beta_{n} y}-\tilde{B}_{c_{1}, n} e^{-j \beta_{n} y}\right) \sin \frac{n \pi x}{x_{0}}
$$

and such that $\tilde{\psi}$, taking the place of $\psi$ in (14), satisfies prescribed boundary values on $c_{1}, c_{2}$ and $c_{3}$ (in principle this is only true for $\left.N_{1} \rightarrow \infty\right)$. However, our method uses the combination of three sets of expansion functions, which are, on their own, only sufficient to represent a prescribed boundary value on one of the sides, but altogether on the three sides. Although the introduction of two extra sets of waveguide modes is at first glance unnecessary, our method has the advantage that we can enforce $\psi^{(1)}$ to be zero at $p_{3}$, in this way avoiding the exponential terms in (18) to become large (and analogously for $\psi^{(2)}$ and $\left.\psi^{(3)}\right)$. If, instead, (21) would be used, the exponential behavior of the parallel-plate 
waveguide modes in the direction perpendicular to $c_{1}$ would lead to ill-conditioning and completely inaccurate results.

As for each boundary excitation there exists a unique solution for the expansion coefficients $A_{c_{i}, n}$, it should be possible to construct a set of equations that can be solved directly for these coefficients. This procedure needs to be repeated as many times as there are discretization segments, and would lead to very long calculations. Therefore, the authors have opted for an iterative approach to determine the unknown entries of the DtN matrix. This method has two major advantages. First of all, it is possible to construct a very good initial guess to start the iteration (assuming at first there is no interaction between the sides), and furthermore, the iterative method has an exponential convergence behavior (as explained in the sequel), which leads to an accurate solution within a very limited number of iteration steps.

\section{B. Discretization of $\psi_{c}$ and Iterative Procedure}

The following paragraphs describe the iterative prodedure to determine the expansion coefficients $A_{c_{i}, n}$ of (14-17), which in the sequel will be called the Iterative Combined Waveguide Modes (ICWM) algorithm. Before giving some more mathematical details, we start with a brief physical description of the method. On each side, the unknown function $\psi$ is first expanded in a set of non-uniform basis functions, typically pulses or hat functions. The complex amplitudes of these functions are collected in a column vector $\Psi_{c}$. Next, this representation is recast in the form (15-18) necessitating the introduction of a mapping matrix $\mathrm{W}$ between $\Psi_{\mathrm{c}}$ and the $A$ coeffcients in (15-17) collected in the column vector $\mathrm{A}$. To be able to determine $W$, three additional matrix operations are needed, i.e. $Q, D$ and $P$. D and $Q$ transform the original basis expansion on each side into Fourier series expansions. Then $\mathrm{P}$ matrices are defined that project the Fourier series coefficients from one side of the triangle onto another side. These matrices account for the "overlap", i.e. for the way in which the three sets of parallel-plate waveguide modes influence each other. For a better readibility of this text, the explicit form of several of these matrices is not given, but can be found in the Appendix.

The proposed method is based on improving an initial guess for the expansion coefficients, by cycling through the sides untill the required accuracy is reached. Suppose we start from side $c_{1}$. The expansion of $\psi_{c_{1}}$ in sine functions yields a first approximation $\psi^{(1),\langle 1\rangle}$ for $\psi^{(1)}$. The notation $\langle n\rangle$ will be used to denote a certain value after iteration cycle $n$. Subtracting the contribution of $\psi^{(1),\langle 1\rangle}$ on $c_{2}$ from the actual boundary value $\psi_{c_{2}}$, and expanding this result in sines, i.e., calculating the coefficients $\left\{A_{c_{2}}^{\langle 1\rangle}\right\}$, yields $\psi^{(2),\langle 1\rangle}$. For the third side, the contributions of both $\psi^{(1),\langle 1\rangle}$ and $\psi^{(2),\langle 1\rangle}$ are subtracted from $\psi_{c_{3}}$, before expanding it into sines, yielding $\psi^{(3),\langle 1\rangle}$. This is the end of the first iteration cycle. From now on, both previous contributions from the expansions on two of the sides to the third side are subtracted from the actual boundary value, and the remainder is expanded into sines. In each iteration cycle, the contribution of the three $\psi^{(i),\langle n\rangle}$ on each of the sides constitute a continually better approximation of the actual boundary value $\psi_{c_{i}}$. In Section IV, the convergence properties of the method are numerically illustrated. We now first go into some of the mathematical details.

In order to discretize the function $\psi$ on side $c_{1}$, the side is divided into segments, using a number of discretization points $x_{m},\left(m=1, \ldots, M_{1}+1\right)$, with $x_{1}$ and $x_{M_{1}+1}$ the corner points. A uniform distribution of these points along the sides is not required. They can be chosen at will, so as to assure an optimal representation of the continuous function $\psi_{c_{1}}$. The normalized discretization points $s_{m}$ on side $c_{1}$ are defined by $x_{m}=s_{m} l_{1}$, and analogously on the other sides. With these, we get for side $c_{i}$ (with $i=1,2,3$ )

$$
\psi_{c_{i}}(s) \simeq \sum_{m=1}^{M_{i}} \Psi_{c_{i}, m} t_{c_{i}, m}(s), \quad 0 \leq s \leq 1 .
$$

The functions $t_{c_{i}, m}(s)$, with $m=1, \ldots, M_{i}$, can be a constant pulse on the interval $\left[s_{m}, s_{m+1}\right]$ (corresponding, e.g on $c_{1}$, to $\left.x \in\left[x_{m}, x_{m+1}\right]\right)$, a piecewise linear 'hat' function on $\left[s_{m-1}, s_{m+1}\right]$, or any other basis function.

The coefficients $\Psi_{c_{i}, m}$ are taken together into one column vector $\Psi_{c}$ as

$$
\Psi_{\mathrm{c}}=\left[\begin{array}{l}
\Psi_{\mathrm{c}_{1}} \\
\Psi_{\mathrm{c}_{2}} \\
\Psi_{\mathrm{c}_{3}}
\end{array}\right]
$$

in which $\left[\Psi_{c_{\mathrm{i}}}\right]_{m}=\Psi_{c_{i}, m}$. The expansion coefficients $A_{c_{i}, n}$ from (15-17) are taken together into column vectors $A_{c_{i}}$. The purpose of the ICWM algorithm is the determination of the $N_{i} \times M$ matrices $\mathrm{W}_{\mathrm{c}_{\mathrm{i}}}$ (with $M=M_{1}+M_{2}+M_{3}$ ), defined by

$$
A_{c_{i}}=W_{c_{i}} \Psi_{c} .
$$

Once these matrices are known, the expansion (14-17) is fully determined for any boundary function $\psi_{c}$, and $\partial \psi / \partial n$ can be determined from the normal derivative of the functions $f_{c_{1}, n}$, $f_{c_{2}, n}$, and $f_{c_{3}, n}$.

In order to calculate the matrices $W_{c_{i}}$, two different types of interactions have to be worked out. On the one hand, we need to transform the coefficients of a discretized function on $c_{i}$ into its sine expansion. On the other hand, we need to determine the expansion coefficients that result from expanding subfunction $\psi^{(j)}$, but evaluated on side $c_{i}(i \neq j)$, into sines on side $c_{i}$.

In order to simplify the notations used in the sequel, some auxiliary functions are defined here, related to the evaluation of $f_{c_{1}}(x, y)$ and its derivatives on sides $c_{2}$ and $c_{3}$.

$$
\begin{aligned}
\gamma_{n}^{-}(s) & \stackrel{\text { def }}{=}\left(e^{j \beta_{n} y_{0}(1-s)}-e^{j \beta_{n} y_{0}(1+s)}\right) \\
\gamma_{n}^{+}(s) & \stackrel{\text { def }}{=}\left(e^{j \beta_{n} y_{0}(1-s)}+e^{j \beta_{n} y_{0}(1+s)}\right) \\
\zeta_{2, n}(s) & \stackrel{\text { def }}{=} \frac{n \pi}{x_{0}}\left(x_{0}+s\left(x_{1}-x_{0}\right)\right) \\
\zeta_{3, n}(s) & \stackrel{\text { def }}{=} \frac{n \pi}{x_{0}}\left((1-s) x_{1}\right)
\end{aligned}
$$

These functions will be used frequently in the sequel, without each time referring to (25-28), though. For example, $\psi^{(1)}$ evaluated on $c_{3}$ can now be compactly written as

$$
\psi_{c_{3}}^{(1)}(s)=\sum_{n=1}^{N_{1}} A_{c_{1}, n} \gamma_{n}^{-}(s) \sin \zeta_{3, n}(s) .
$$


First, the transformation matrices $Q_{i}$ and the scaling matrices $D_{i}$ are defined. They transform the coefficients $\Psi_{c_{i}, m}^{(i)}$, with the superscript $(i)$ indicating the contribution of $\psi^{(i)}$ only, into its sine expansion coefficients $A_{c_{i}}$. The expansion of $\psi^{(1)}$ on side $c_{1}$,

$$
\begin{aligned}
\psi_{c_{1}}^{(1)}(s) & \simeq \sum_{m=1}^{M_{1}} \Psi_{c_{1}, m}^{(1)} t_{c_{1}, m}(s) \\
& \simeq \sum_{n=1}^{N_{1}} A_{c_{1}, n} \gamma_{n}^{-}(1) \sin n \pi s
\end{aligned}
$$

is weighted with the set $\left\{2 \sin n \pi s, n=1, \ldots, N_{1}\right\}$. Taking the coefficients together in the vectors $\Psi_{c_{1}}^{(1)}$ and $A_{c_{1}}$, leads to

$$
\mathrm{Q}_{1} \Psi_{\mathrm{c}_{1}}^{(1)}=\mathrm{D}_{1}^{-1} \mathrm{~A}_{\mathrm{c}_{1}}
$$

with the explicit form of the $N_{1} \times M_{1}$ matrix $\mathrm{Q}_{1}$ and the $N_{1} \times N_{1}$ diagonal matrix $\mathrm{D}_{1}$ given in the Appendix.

For the other sides $c_{2}$ and $c_{3}$, the primed and double primed quantities can be used, as indicated in (19) and (20). We schematically write this as

$$
\begin{array}{ll}
\mathrm{Q}_{2}=\mathrm{Q}_{1}^{\prime}, & \mathrm{D}_{2}=\mathrm{D}_{1}^{\prime}, \\
\mathrm{Q}_{3}=\mathrm{Q}_{1}^{\prime \prime}, & \mathrm{D}_{3}=\mathrm{D}_{1}^{\prime \prime} .
\end{array}
$$

The expansion matrices $\mathrm{P}_{\mathrm{ij}}$ are used to calculate the coefficients $C_{c_{i}, n}^{(j)}$ in the sine expansion on $c_{i}$ of subfunction $\psi^{(j)}$, defined by its coefficients $A_{c_{j}, n}$. Evaluating $\psi^{(1)}$ on $c_{2}$ and on $c_{3}$ and expanding it into sines on these sides, yields, with (11), (12), (15) and (18),

$$
\begin{aligned}
\psi_{c_{2}}^{(1)}(s) & \simeq \sum_{n_{2}=1}^{N_{2}} C_{c_{2}, n_{2}}^{(1)} \sin n_{2} \pi s \\
& \simeq \sum_{n_{1}=1}^{N_{1}} A_{c_{1}, n_{1}} \gamma_{n_{1}}^{-}(1-s) \sin \zeta_{2, n_{1}}(s) \\
\psi_{c_{3}}^{(1)}(s) & \simeq \sum_{n_{3}=1}^{N_{3}} C_{c_{3}, n_{3}}^{(1)} \sin n_{3} \pi s \\
& \simeq \sum_{n_{1}=1}^{N_{1}} A_{c_{1}, n_{1}} \gamma_{n_{1}}^{-}(s) \sin \zeta_{3, n_{1}}(s) .
\end{aligned}
$$

Weighting (35) and (36) with the set $\left\{2 \sin n_{2} \pi s\right\}$, and (37) and (38) with the set $\left\{2 \sin n_{3} \pi s\right\}$, yields

$$
\begin{aligned}
& C_{c_{2}}^{(1)}=P_{21} A_{c_{1}} \\
& C_{c_{3}}^{(1)}=P_{31} A_{c_{1}}
\end{aligned}
$$

with the $N_{2} \times N_{1}$ matrix $\mathrm{P}_{21}$ and the $N_{3} \times N_{1}$ matrix $\mathrm{P}_{31}$ specified in the Appendix.

Performing the same operations for the rotated geometries shown in Fig. 2 (b) and (c), leads to the other required matrices

$$
\begin{array}{ll}
\mathrm{P}_{12}=\mathrm{P}_{31}^{\prime}, & \mathrm{P}_{32}=\mathrm{P}_{21}^{\prime} \\
\mathrm{P}_{13}=\mathrm{P}_{21}^{\prime \prime}, & \mathrm{P}_{23}=\mathrm{P}_{31}^{\prime \prime} .
\end{array}
$$

The ICWM procedure as outlined above is an iterative procedure to determine the $W_{c_{i}}$ matrices. Initially, all three matrices are supposed zero. We now describe cycle $n$ of the iteration. Suppose after $n-1$ iteration cycles, we want to determine the coefficients $A_{c_{1}}^{\langle n\rangle}$ from $A_{c_{2}}^{\langle n-1\rangle}$ and $A_{c_{3}}^{\langle n-1\rangle}$. Evaluation of (14) with the LHS discretized using (22), evaluated on $c_{1}$ with (10), and with the insertion of expansion (15) and (18), gives

$$
\begin{aligned}
& \sum_{m=1}^{M_{1}} \Psi_{c_{1}, m} t_{c_{1}, m}(s)-\left(\psi_{c_{1}}^{(2),\langle n-1\rangle}+\psi_{c_{1}}^{(3),\langle n-1\rangle}\right) \\
& =\sum_{n=1}^{N_{1}} A_{c_{1}, n}^{\langle n\rangle} \gamma_{n}^{-}(1) \sin n \pi s .
\end{aligned}
$$

Weighting (43) with the set $\{2 \sin n \pi s\}$ yields

$$
\mathrm{Q}_{1} \Psi_{\mathrm{c}_{1}}-\left(\mathrm{C}_{\mathrm{c}_{1}}^{(2),\langle n-1\rangle}+\mathrm{C}_{\mathrm{c}_{1}}^{(3),\langle n-1\rangle}\right)=\mathrm{D}_{1}{ }^{-1} \mathrm{~A}_{\mathrm{c}_{1}}^{\langle n\rangle} .
$$

The analogous relationships as (39), but involving $P_{12}$ and $\mathrm{P}_{13}$, allow to write (44) as

$$
A_{c_{1}}^{\langle n\rangle}=D_{1}\left(Q_{1} \Psi_{c_{1}}-P_{12} A_{c_{2}}^{\langle n-1\rangle}-P_{13} A_{c_{3}}^{\langle n-1\rangle}\right) .
$$

Define the matrix

$$
\tilde{\mathrm{Q}}_{1}=\left[\mathrm{Q}_{1}, 0_{\mathrm{N}_{1} \times \mathrm{M}_{2}}, 0_{\mathrm{N}_{1} \times \mathrm{M}_{3}}\right],
$$

using the notation $0_{\mathrm{N}_{\mathrm{i}} \times \mathrm{M}_{\mathrm{j}}}$ for a $N_{i} \times M_{j}$ zero matrix. Inserting the relationships (24) leads from (45) to

$$
\mathrm{W}_{\mathrm{c}_{1}}^{\langle n\rangle}=\mathrm{D}_{1}\left(\tilde{\mathrm{Q}}_{1}-\mathrm{P}_{12} \mathrm{~W}_{\mathrm{c}_{2}}^{\langle n-1\rangle}-\mathrm{P}_{13} \mathrm{~W}_{\mathrm{c}_{3}}^{\langle n-1\rangle}\right) .
$$

Analogous calculations show that

$$
\begin{aligned}
& \mathrm{W}_{\mathrm{c}_{2}}^{\langle n\rangle}=\mathrm{D}_{2}\left(\tilde{\mathrm{Q}}_{2}-\mathrm{P}_{21} \mathrm{~W}_{\mathrm{c}_{1}}^{\langle n\rangle}-\mathrm{P}_{23} \mathrm{~W}_{\mathrm{c}_{3}}^{\langle n-1\rangle}\right) \\
& \mathrm{W}_{\mathrm{c}_{3}}^{\langle n\rangle}=\mathrm{D}_{3}\left(\tilde{\mathrm{Q}}_{3}-\mathrm{P}_{31} \mathrm{~W}_{\mathrm{c}_{1}}^{\langle n\rangle}-\mathrm{P}_{32} \mathrm{~W}_{\mathrm{c}_{2}}^{\langle n\rangle}\right)
\end{aligned}
$$

with

$$
\begin{array}{ll}
\mathrm{D}_{2}=\mathrm{D}_{1}^{\prime}, & \tilde{\mathrm{Q}}_{2}=\left[0_{\mathrm{N}_{2} \times \mathrm{M}_{1}}, \mathrm{Q}_{2}, 0_{\mathrm{N}_{2} \times \mathrm{M}_{3}}\right] \\
\mathrm{D}_{3}=\mathrm{D}_{1}^{\prime \prime}, & \tilde{\mathrm{Q}}_{3}=\left[0_{\mathrm{N}_{3} \times \mathrm{M}_{1}}, 0_{\mathrm{N}_{3} \times \mathrm{M}_{2}}, \mathrm{Q}_{3}\right] .
\end{array}
$$

The update equations (47), (48) and (49) form the core of the $n$ 'th iteration cycle of the ICWM procedure.

\section{Normal Derivative Calculation}

The outward pointing normal derivative $\partial \psi_{c} / \partial n$ is discretized on side $i$ as

$$
\frac{\partial \psi_{c_{i}}(s)}{\partial n} \simeq \sum_{m=1}^{M_{i}} \Gamma_{c_{i}, m} t_{c_{i}, m}(s) .
$$

The purpose is to determine the $\Gamma_{c_{i}, m}$ coefficients, taken together per side $i$ into the vector $\Gamma_{\mathrm{c}_{\mathrm{i}}}$, such that

$$
\Gamma_{\mathrm{c}_{\mathrm{i}}}=\sum_{j=1}^{3} \Gamma_{\mathrm{c}_{\mathrm{i}}}^{(\mathrm{j})}=\sum_{j=1}^{3} \mathrm{~T}_{\mathrm{ij}} \mathrm{A}_{\mathrm{c}_{\mathrm{j}}}
$$

in which $\Gamma_{\mathrm{c}_{\mathrm{i}}}^{(\mathrm{j})}$ contains the coefficients $\Gamma_{c_{i}}^{(j)}$ in the contribution from $\psi^{(j)}$ to the total normal derivative on $c_{i}$. Hence, with (24) and by grouping the vectors $\Gamma_{c_{i}}$ into $\Gamma_{c}$, we find

$$
\Gamma_{\mathrm{c}}=\mathrm{D} \Psi_{\mathrm{c}}
$$

with

$$
D=\left[\begin{array}{l}
T_{11} W_{c_{1}}+T_{12} W_{c_{2}}+T_{13} W_{c_{3}} \\
T_{21} W_{c_{1}}+T_{22} W_{c_{2}}+T_{23} W_{c_{3}} \\
T_{31} W_{c_{1}}+T_{32} W_{c_{2}}+T_{33} W_{c_{3}}
\end{array}\right]
$$


which is the discretized form of (8), i.e., the matrixrepresentation of the DtN operator, mapping $\psi_{c}$ onto $\partial \psi_{c} / \partial n$.

The matrices $T_{i j}$ are determined by calculating the outward normal derivative of $\psi^{(j)}$ on side $c_{i}$, and weighting the result with the basis functions $t_{c_{i}}(s)$ along that side.

On $c_{1}, \partial \psi^{(1)} / \partial n$ is written as

$$
\begin{aligned}
\frac{\partial \psi_{c_{1}}^{(1)}(s)}{\partial n} & \simeq \sum_{m=1}^{M_{1}} \Gamma_{c_{1}, m}^{(1)} t_{c_{1}, m}(s) \\
& \simeq-\sum_{n=1}^{N_{1}} A_{c_{1}, n} j \beta_{n} \gamma_{n}^{+}(1) \sin n \pi s
\end{aligned}
$$

and leads to

$$
\Gamma_{\mathrm{c}_{1}}^{(1)}=\mathrm{T}_{11} \mathrm{~A}_{\mathrm{c}_{1}} \text {. }
$$

On $c_{2}$, the normal derivative of $\psi^{(1)}$ becomes, with (13),

$$
\begin{aligned}
\frac{\partial \psi_{c_{2}}^{(1)}(s)}{\partial n}= & \mathbf{u}_{2} \cdot\left[\nabla \psi^{(1)}\right]_{c_{2}} \\
\simeq & \sum_{m=1}^{M_{2}} \Gamma_{c_{2}, m}^{(1)} t_{c_{2}, m}(s) \\
\simeq & \sum_{n=1}^{N_{1}} A_{c_{1}, n}\left(\frac{y_{0}}{l_{2}} \gamma_{n}^{-}(1-s) \frac{n \pi}{x_{0}} \cos \zeta_{2, n}(s)\right. \\
& \left.+\frac{x_{0}-x_{1}}{l_{2}} j \beta_{n} \gamma_{n}^{+}(1-s) \sin \zeta_{2, n}(s)\right) .
\end{aligned}
$$

Weighting (60) and (61) with the basis functions $t_{c_{2}, m}(s)$ on $c_{2}$ leads to

$$
\Gamma_{\mathrm{c}_{2}}^{(1)}=\mathrm{T}_{21} \mathrm{~A}_{\mathrm{c}_{1}} \text {. }
$$

Analogously, $\partial \psi_{c_{3}}^{(1)} / \partial n$ becomes

$$
\begin{aligned}
\frac{\partial \psi_{c_{3}}^{(1)}(s)}{\partial n} & =\mathbf{u}_{3} \cdot\left[\nabla \psi^{(1)}\right]_{c_{3}} \\
& \simeq \sum_{m=1}^{M_{3}} \Gamma_{c_{3}, m}^{(1)} t_{c_{3}, m}(s) \\
& \simeq \sum_{n=1}^{N_{1}} A_{c_{1}, n}\left(-\frac{y_{0}}{l_{3}} \gamma_{n}^{-}(s) \frac{n \pi}{x_{0}} \cos \zeta_{3, n}(s)\right. \\
& \left.\quad+\frac{x_{1}}{l_{3}} j \beta_{n} \gamma_{n}^{+}(s) \sin \zeta_{3, n}(s)\right)
\end{aligned}
$$

and leads to

$$
\Gamma_{\mathrm{c}_{3}}^{(1)}=\mathrm{T}_{31} \mathrm{~A}_{\mathrm{c}_{1}} \text {. }
$$

The $M_{i} \times N_{1}$ matrices $\mathrm{T}_{\mathrm{i} 1}$ are defined in the Appendix. For the remaining $T_{i j}$ matrices, we can immediately write

$$
\begin{array}{lll}
\mathrm{T}_{12}=\mathrm{T}_{31}^{\prime}, & \mathrm{T}_{22}=\mathrm{T}_{11}^{\prime}, & \mathrm{T}_{32}=\mathrm{T}_{21}^{\prime} \\
\mathrm{T}_{13}=\mathrm{T}_{21}^{\prime \prime}, & \mathrm{T}_{23}=\mathrm{T}_{31}^{\prime \prime}, & \mathrm{T}_{33}=\mathrm{T}_{11}^{\prime \prime} .
\end{array}
$$

\section{Elimination of THE GibBs EFFECT}

For the non-differential DtN operator as defined by (8), the Gibbs phenomena at the corners of the triangle are considerable, due to the expansion functions $f_{c_{i}, n}$ which are zero in the corner points, and hence not apt to represent a non-zero corner value.
As will become clear in Section IV from the numerical data, this Gibbs phenomenon corrupts the solution along the complete boundary. An accurate elimination of the Gibbs effect is therefore required for an arbitrary value of $k^{2}$. The solution for the Gibbs effect presented in [11] for a rectangular cross-section, only deals with $k^{2}=0$. Below, a generalization is presented. This generalization is only valid provided the boundary value of $\psi_{c}$ is continuous, but this is indeed the case, both for the scalar electric potential and for the longitudinal electric field.

The DtN operator (55) is only correct provided all corner values are zero. A function satisfying this requirement is obtained by subtracting from $\psi$ three analytically known functions $\hat{\psi}^{p_{i}}$, that satisfy (9), have a non-zero corner value at $p_{i}$, and are zero on the other corners. We start at corner $p_{1}$. The proposed function $\hat{\psi}^{p_{1}}$ is defined by

$$
\hat{\psi}^{p_{1}}(x, y)=\hat{\alpha}^{p_{1}} \cos \frac{\pi x}{2 x_{0}}\left(e^{j \hat{\beta} y}-e^{j \hat{\beta}\left(2 y_{0}-y\right)}\right),
$$

with $\hat{\beta}^{2}=k^{2}-\left(\pi / 2 x_{0}\right)^{2}$. It reaches its maximal amplitude at $p_{1}$, and is, as required, zero at $p_{2}$ and $p_{3}$. Analogous functions are defined, associated with $p_{2}$ and $p_{3}$. We now have to determine a matrix $\hat{D}^{p_{1}}$, which transforms $\Psi_{c}$ into the normal derivative of $\hat{\psi}^{p_{1}}$, with a correctly determined coefficient $\hat{\alpha}^{p_{1}}$. Secondly, a matrix $\hat{\mathrm{R}}^{\mathrm{p}_{1}}$ is needed, to reduce the original boundary coefficients $\Psi_{c}$ to those without the contribution of $\hat{\psi}^{p_{1}}$. The same argumentation can be followed to treat corners $p_{2}$ and $p_{3}$, with the introduction of analogous matrices. This results in

$$
\Gamma_{\mathrm{c}}=\mathrm{D}_{\mathrm{tot}} \Psi_{\mathrm{c}}
$$

with

$$
D_{\text {tot }}=\left(D \hat{R}^{p_{3}} \hat{R}^{p_{2}} \hat{R}^{p_{1}}+\hat{D}^{p_{3}} \hat{R}^{p_{2}} \hat{R}^{p_{1}}+\hat{D}^{p_{2}} \hat{R}^{p_{1}}+\hat{D}^{p_{1}}\right)
$$

Compared to the original discretized form D (55) of the DtN operator, $D_{\text {tot }}$ is its modified form which does no longer suffer from the Gibbs phenomenon. The expressions for $\hat{D}^{p_{i}}$ and $\hat{R}^{p_{i}}$ ( $i=1,2,3)$ can be found in the Appendix.

\section{NuMERICAL RESUlTS}

A few numerical simulations are presented, to investigate the convergence and accuracy properties of the method, including an illustration of the effectiveness of the Gibbs phenomenon elimination as described in Section III. In a few further examples, the inductive and resistive behavior of trapezoidal conductors is investigated. All simulations were done with a uniform, piecewise constant approximation of the longitudinal electric field on the triangles, except for the last one, where we used a piecewise linear discretization on the boundaries.

\section{A. Numerical Accuracy and Convergence Properties of the Method}

In order to illustrate the effectiveness of the method described in Section III to eliminate the Gibbs effect at the triangles' corners, we compare the normal derivative $\partial \psi / \partial n$ of 
a function $\psi(x, y)$, along the boundary of a triangle $T_{1}$ without and with the use of the correction formula (71) instead of (55).

Triangle $T_{1}$ is shown in the inset of Fig. 3 (a), and has side lengths of, respectively, $c_{1}=4 \mathrm{~mm}$ and $c_{2}=c_{3}=2.5$ $\mathrm{mm}$. The boundary value $\psi_{c}$ is chosen to be continuous along its boundary, and linear on each side, with corner values $\psi_{p_{1}}=0, \psi_{p_{2}}=-1$, and $\psi_{p_{3}}=1$. For a high resolution of the displayed results along the boundary, the number of discretization intervals is chosen to be 300 along $c_{1}$, and 188 along $c_{2}$ and $c_{3}$. Obviously, for most applications the results will be accurate enough with a coarser discretization. The number of sine functions used along each side amounts to 400 along $c_{1}$, and 250 along $c_{2}$ and $c_{3}$.

Fig. 3 (a) displays the results for the dielectric case (neglecting the displacement currents in the quasi-TM case), with $\psi$ satisfying Laplace's equation in $T_{1}$. Without the Gibbs effect compensation, i.e., using only (55), the oscillations are huge and not even restricted to the corner areas. Note that corner $p_{1}$ does not introduce any Gibbs effect, because $\psi_{c}$ is exactly zero at $p_{1}$. With (71), the numerical result is almost indistinguishable from the exact $\partial \psi / \partial n$, namely -1000 on $c_{1}$, 650 on $c_{2}$ and 950 on $c_{3}$. An analogous comparison is made in Fig. 3 (b), for the same boundary value $\psi_{c}$, but with $\psi$ satisfying the diffusion equation (4) in $T_{1}$, for a conductivity $\sigma=57.2 \mathrm{MS} / \mathrm{m}$ and at $100 \mathrm{kHz}$. The Gibbs effect is especially strong near the corners now, but again totally eliminated by using (71).

A peculiarity of the results in Fig. 3 (b) is the behavior near the corners. This is worth some additional comments, given its general validity and importance for the high-frequency current distribution near an edge. At $100 \mathrm{kHz}$, the skin depth $\delta \approx 0.21 \mathrm{~mm}$. Based on a local plane wave approximation, $\psi$ will be exponentially damped, proportional to $e^{-n / \delta}$, with $n$ the coordinate in the normal direction $n$ from a boundary point $p$ towards the inside of the triangle. This approximation does not hold, if the distance along $n$ to the opposite side is smaller than a few times the skin depth. If, e.g., $p$ lays on $c_{1}$ at a distance $\delta / 2$ from the corner point $p_{2}$, then the distance from $p$ to the adjacent side $c_{2}$ (in the normal direction with respect to $c_{1}$ ) is $3 \delta / 8$ (for a corner of $36.9^{\circ}$ ). If $p$ starts to approach $p_{2}$ even closer, the diffusion term no longer plays a role, and we should therefore get the same result as in Fig. 3 (a). The boundary interval of length $\delta$ centered around $p_{2}$ is indicated by the dashed vertical lines in Fig. 3 (b). The normal derivative $\partial \psi / \partial n$ in this interval is indeed very similar to the corresponding solution of Fig. 3 (a), indicated in dash-dot lines. This phenomenon is clearly visible for the sharp corners $p_{1}$ and $p_{2}$. For the obtuse corner $p_{3}$ however, this is not the case. The reason for that is, that for any boundary point $p$ close to $p_{3}$, the diffusion term still plays a role, because in the normal direction, the adjacent side is not reached, let alone that this distance becomes $\ll \delta$ near the corner.

In a second numerical experiment, the convergence of the iterative method is investigated. As explained in Section II-C, the normal derivative $\partial \psi / \partial n$ is determined analytically from the expansion of $\psi$ itself. The correctness of $\partial \psi / \partial n$ is hence limited by the accuracy of the expansion of $\psi$ in $T_{1}$ and more specifically on its boundary, as each term in the

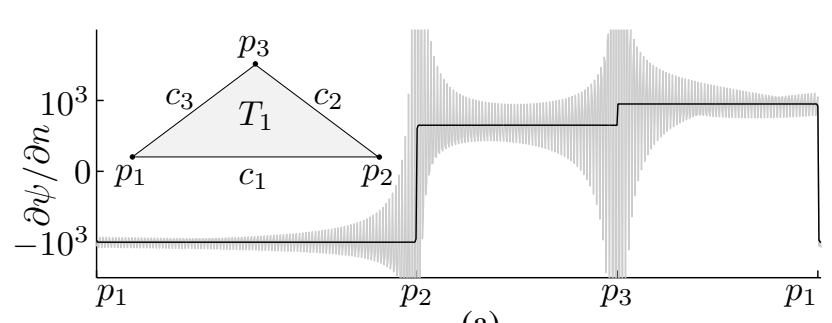

(a)

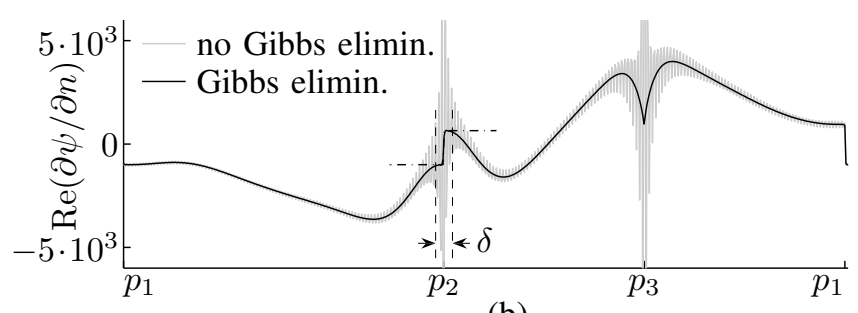

(b)

Fig. 3. Normal derivative $\partial \psi / \partial n$ along the boundary of triangle $T_{1}$ (see inset of (a), $c_{1}=4 \mathrm{~mm}, c_{2}=c_{3}=2.5 \mathrm{~mm}$ ) both without and with elimination of the Gibbs effect, for $\psi$ linear along the sides of $T_{1}$, and with $\psi_{p_{1}}=0$, $\psi_{p_{2}}=-1, \psi_{p_{3}}=1$. The simulations were performed at $100 \mathrm{kHz}$, for (a) $T_{1}$ as a dielectric, and (b) $T_{1}$ as a conductor with $\sigma=57.2 \mathrm{MS} / \mathrm{m}$, with only $\operatorname{Re}(\partial \psi / \partial n)$ shown.

expansion exactly satisfies the governing equation (9) inside $T_{1}$. Therefore, it is investigated how the boundary value $\psi_{c}^{(n)}$ of the expansion after $n$ iteration steps becomes a better approximation of the exact $\psi_{c}$ for increasing $n$. Again consider the function $\psi(x, y)$ over triangle $T_{1}$, but now with a constant boundary value $\psi_{c}=\psi_{0}$. Fig. 4 displays the relative error of $\psi_{c}^{\langle n\rangle}$ with respect to $\psi_{0}$, for (a) $\psi$ satisfying Laplace's equation, and (b) the diffusion equation, as in Fig. 3. As for $\partial \psi / \partial n$, the Gibbs effect in the calculation of $\psi_{c}$ is taken care of by subtracting an analytical part with the same corner values as $\psi_{c}$, and then using the matrices $\mathrm{W}_{c_{1}}^{\langle n\rangle}, \mathrm{W}_{c_{2}}^{\langle n\rangle}$, and $\mathrm{W}_{c_{3}}^{\langle n\rangle}$ to calculate the expansion coefficients for the sine expansions on each side of the remaining part of $\psi$, which now has zero corner values.

It is clearly visible in both Fig. 4 (a) and (b) that the error rapidly decreases with each iteration step. After a certain number of iteration steps (about 8 in this case, and earlier on side $c_{2}$ and $c_{3}$ ), the relative error will no longer further decrease (but is already much smaller than 1/1000). This is not due to the limited accuracy of the expansion coefficients, as will be shown in a further numerical experiment. It is caused by the limited accuracy in the estimation of the corner values of $\psi$, that are further processed for Gibbs effect elimination.

The convergence behavior of the iterative procedure to find the expansion of $\psi$ depends much stronger on the triangle's shape than on the diffusion coefficient $k^{2}$ in (9). It is expected that the scheme converges slower, the sharper a corner of the triangle becomes, due to the increased interaction between the corresponding adjacent sides. Consider the triangular conductor $T_{2}$ (shown in the inset of Fig. 5), with conductivity $\sigma=57.2 \mathrm{MS} / \mathrm{m}$., and at $100 \mathrm{kHz}$. The area of the isosceles triangle $T_{2}$ is kept to $1 \mathrm{~mm}^{2}$, whereas the top angle is 


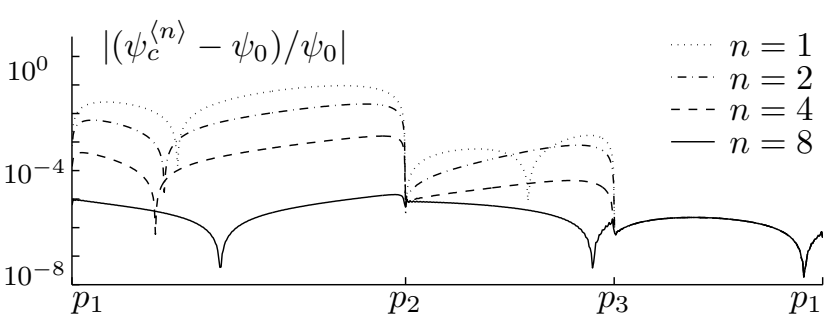

(a)

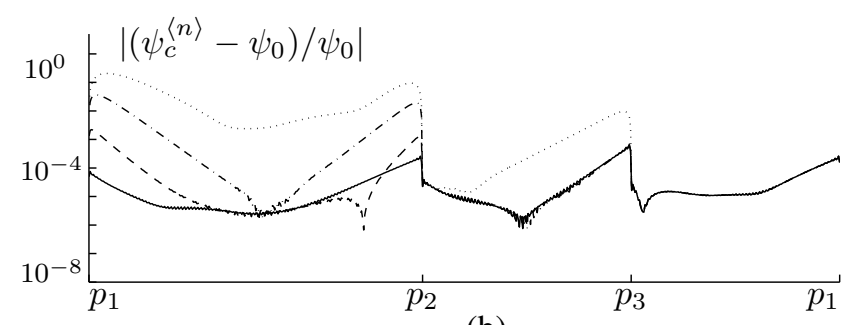

(b)

Fig. 4. Relative error after $n$ iterations, in the estimation $\psi_{c}^{\langle n\rangle}$ of a constant boundary value $\psi_{c}=\psi_{0}$, for triangle $T_{1}$ (see Fig. 3 (a)). The simulations were performed at $100 \mathrm{kHz}$, for (a) $T_{1}$ as a dielectric, and (b) $T_{1}$ as a conductor with $\sigma=57.2 \mathrm{MS} / \mathrm{m}$.

varied from $60^{\circ}$ to $3.75^{\circ}$. The convergence of matrix $\mathrm{W}_{c_{2}}^{\langle n\rangle}$ is presented in Fig. 5 by means of the normalized Frobenius norm of the difference between two consecutive matrices $\mathrm{W}_{c_{2}}^{\langle n-1\rangle}$ and $\mathrm{W}_{c_{2}}^{\langle n\rangle}$, i.e., by

$$
\frac{\left\|\mathrm{W}_{c_{2}}^{\langle n\rangle}-\mathrm{W}_{c_{2}}^{\langle n-1\rangle}\right\|}{\left\|\mathrm{W}_{c_{2}}^{\langle n\rangle}\right\|}=\frac{\sqrt{\sum_{i, j}\left|\left[\mathrm{~W}_{c_{2}}^{\langle n\rangle}\right]_{i, j}-\left[\mathrm{W}_{c_{2}}^{\langle n-1\rangle}\right]_{i, j}\right|^{2}}}{\sqrt{\sum_{i, j}\left|\left[\mathrm{~W}_{c_{2}}^{\langle n\rangle}\right]_{i, j}\right|^{2}}}
$$

For $\alpha=60^{\circ}$, the accuracy is only limited by the floating point precision within less than 20 iteration steps. The smaller $\alpha$ becomes, the slower the iterative procedure converges, but even for $\alpha=3.75^{\circ}$ the difference between steps $n-1$ and $n$ decreases exponentially. In this example we used 228 parallelplate waveguide modes per side.

After these convincing examples of the convergence behavior of the ICWM algorithm, the authors would like to add a few comments to indicate that the scheme will always converge. This is indeed the case, at least within the validity range of the quasi-TM analysis. The reason is, that $\operatorname{Re}\left(j \beta_{n}\right)$, with $\beta_{n}$ defined as for (18), is strictly negative. The function $f_{c_{1}, n}$ will consequently only have a small contribution to sides $c_{2}$ and $c_{3}$, compared to its function value on $c_{1}$. Generally speaking, a correction of the coefficients $A_{c_{i}, n}$ of the functions $f_{c_{i}, n}$ of side $c_{i}$ will result in a smaller required correction of the coefficients $A_{c_{j}, n}$ on the other sides $c_{j}(j \neq i)$, due to the fact, mentioned above, that $f_{c_{i}, n}$ has only an exponentially small contribution on the other sides $c_{j}(j \neq i)$. As this is true for all sides $i=1,2,3$, the iterative procedure can be expected to converge exponentially and this is what is indeed observed numerically. This explains why, even within the first iteration cycle $n=1$ on Fig. 4, the approximation of $\psi$ on the boundary will be better on side $c_{3}$ than on side $c_{2}$, which

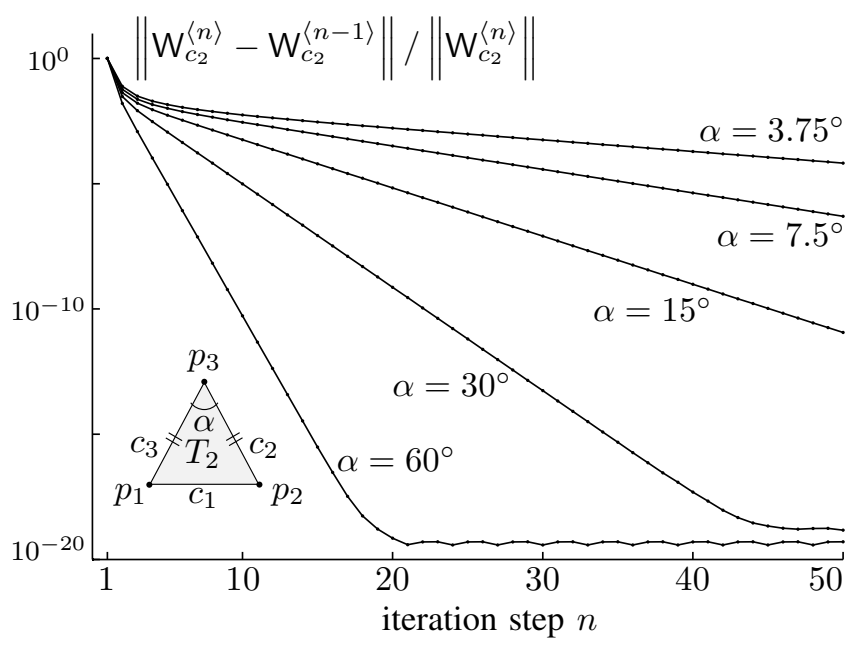

Fig. 5. Convergence behavior of matrix $W_{c_{2}}^{\langle n\rangle}$ as a function of the number of iteration steps $n$ for the isosceles triangle $T_{2}$ with surface $1 \mathrm{~mm}^{2}$ (see inset). Simulations were performed at $100 \mathrm{kHz}$ and with $\sigma=57.2 \mathrm{MS} / \mathrm{m}$.

in turn is better than on $c_{1}$.

A more rigorous convergence analysis could be carried out by investigating the behavior of the matrices $\mathrm{P}_{\mathrm{ij}}$, or by considering the contribution of one expansion function $f_{c_{1}, n}$ on sides $c_{2}$ and $c_{3}$, for the case of the highest possible coupling with side $c_{1}$ (in other words, for the slowest exponential decrease of $f_{c_{1}, n}$ ). This 'worst case scenario' is found for the first order mode $f_{c_{1}, 1}$, for a dielectric material (with $k^{2}=0$ within the quasi-TM approximation), and for sharp corners adjacent to $c_{1}$. Even if the exponential decline is slow and can be approximated by a linear function, the contribution of $f_{c_{1}, 1}$ to $c_{2}$ and $c_{3}$ will still remain small enough to ensure a good convergence, because its function value is forced to zero on $p_{3}$.

\section{B. Characterization of a Single Conductor}

As a verification of the surface admittance matrix for triangles, the p.u.l. resistance of a square copper conductor composed of two triangles is simulated, and compared to data available in literature [7]. The conductor is placed in free space, and has a side length of $4.62 \mathrm{~mm}$ and a conductivity $\sigma=57.2 \mathrm{MS} / \mathrm{m}$. The result is shown in Fig. 6. Exactly the same resistance is obtained with the square conductor composed of two triangles, as the result from [7], determined with the surface admittance matrix of the square. To investigate the influence of the conductor's shape, the resistance of a trapezoid (composed of two triangles, shown in the legend of Fig. 6) and a triangular conductor are shown in Fig. 6 as well. All conductors have the same area, and hence exactly the same low-frequency resistance. At $10 \mathrm{kHz}$, the skin depth $\delta$ in copper is about $0.665 \mathrm{~mm}$, and the major part of the current flows within a layer with thickness $\delta$ underneath the surface. One would therefore expect a resistance, roughly inversely proportional to the circumference, but this is not yet the case within the investigated frequency range. The trapzoid's circumference $L=(2+\sqrt{5}) s$ is higher than the square's 


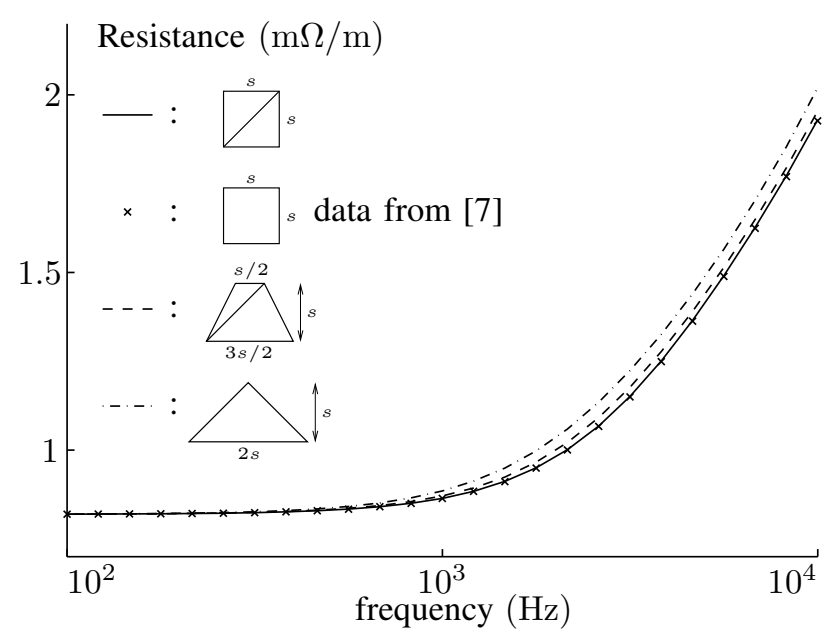

Fig. 6. Resistance $(\mathrm{m} \Omega / \mathrm{m})$ for a square copper conductor $(s=4.62 \mathrm{~mm}$, $\sigma=57.2 \mathrm{MS} / \mathrm{m}$ ) formed by two triangles (solid line) vs. the result from [7] (in x-markers), and compared to a trapezoid (dashed line) and a triangle (dash-dot line) with the same area.

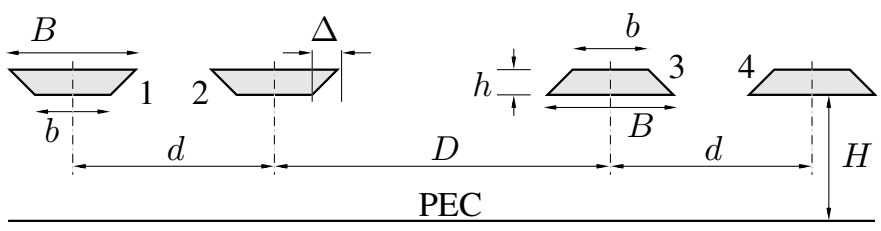

Fig. 7. Structure with 4 trapezoidal copper conductors $(\sigma=57.2 \mathrm{MS} / \mathrm{m})$ above a PEC ground plane (shown on scale). Dimensions are $B=1.5$, $b=0.9, h=0.3, \Delta=0.3, d=2.4, D=4$, and $H=1.5$, all in millimetres.

( $L=4 s)$, yet the latter one has the lowest high-frequency resistance. A similar effect is noticeable for the triangular conductor, with $L=(2+\sqrt{8}) s$ and a still higher resistance. The reason is that the effective length of the skin layer where the current flows, is shortened due to the corner effect at the sharp corners. It is expected that at still higher frequencies, the corner effect becomes less pronounced. A fully detailed investigation of the field behavior at the corners is outside the scope of this paper.

\section{Multiconductor Line with Trapezoidal Conductors}

In a next numerical example, the inductive and resistive characteristics of a multiconductor line are investigated. The purpose is to get a better understanding of the (coupling) behavior of trapezoidal conductors, rather than to simulate a more realistic structure, with a substrate. The structure under investigation is shown in Fig. 7 and consists of two line pairs $(1-2$ and $3-4)$, with an opposite orientation with respect to the nearby perfect electric conducting (PEC) ground plane. All conductors have the conductivity of copper, $\sigma=57.2 \mathrm{MS} / \mathrm{m}$, and the dimensions are indicated in Fig. 7.

The resistance matrix $R$ and the inductance matrix $L$ of the structure are determined over a frequency range from $1 \mathrm{kHz}$ (at with the skin depth $\delta \approx 2 \mathrm{~mm}$ ), up to $100 \mathrm{MHz}$ (where

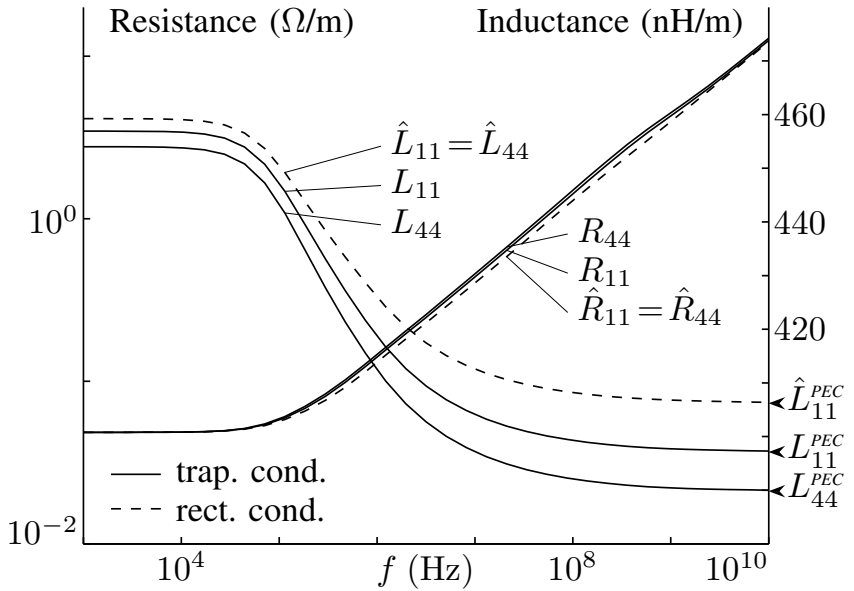

(a)

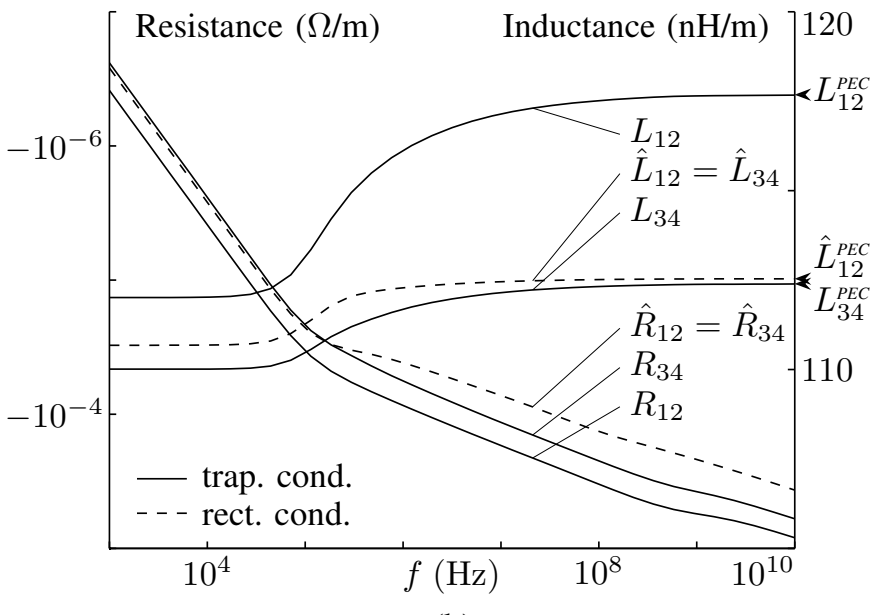

(b)

Fig. 8. Elements of the resistance matrix $R$ and inductance matrix $L$ for the configuration of Fig. 7. Full lines, with trapezoidal conductors; dashed lines, with rectangular conductors. (a) self-inductance and resistance, (b) coupling between the lines of each signal pair $(1-2$, and $3-4)$.

$\delta \approx 0.0067 \mathrm{~mm}$ ). The configuration of Fig. 7 is compared to an analogous configuration with rectangular conductors with the same area (with height $h$, width $(b+B) / 2$, separated by the same distances $d$, resp. $D$, and on the same height $H$ above the ground plane). The results for the trapezoidal conductors are presented in Fig. 8 with full lines, whereas dashed lines are used for the rectangular conductor case. resistance- and inductance-values pertaining to the rectangular case will be denoted by $\hat{R}$ and $\hat{L}$.

Fig. 8 (a) displays the self inductance elements $L_{11}, \hat{L}_{11}$, $L_{44}$ and $\hat{L}_{44}$, and the resistance elements $R_{11}, \hat{R}_{11}, R_{44}$ and $\hat{R}_{44}$. At the lowest frequencies, there is no difference between the resistance elements (as all conductors have the same area), but towards the higher frequencies, the trapezoidal conductors display a higher resistance, in accordance with the result from Fig. 6, except for the highest simulated frequencies, for which the corner effect becomes negligible. The difference between the self-inductance elements are the result of the detailed current distribution in the conductors which are influenced 


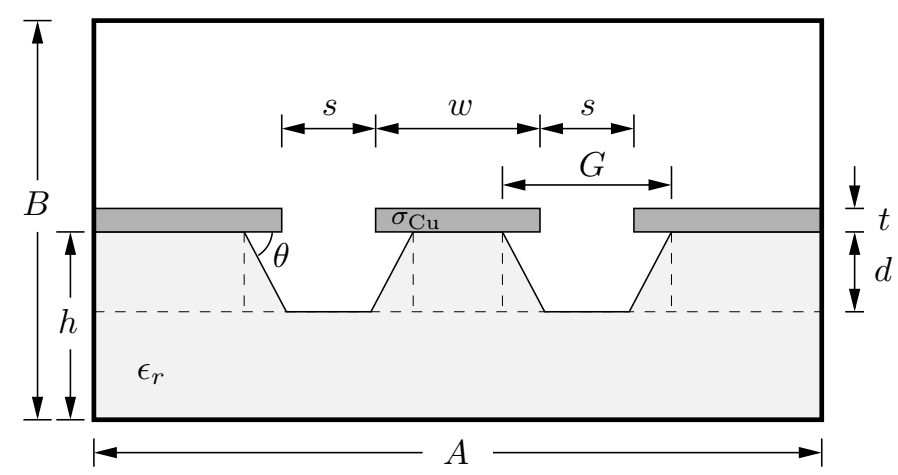

Fig. 9. Coplanar waveguide structure, enclosed by a PEC box (not shown on scale). The dimensions are $A=320, B=500, h=300, w=50$, $s=45, t=1$, all in micrometers. Furthermore, $\theta=54.7^{\circ}, \epsilon_{r}=11.7$, and $\sigma_{\mathrm{Cu}}=57.2 \mathrm{MS} / \mathrm{m}$.

by the corner behavior which is clearly different for sharp and obtuse corners, by the position of these corners w.r.t. the ground plane, and by the proximity effect of the corners. The inductance values for the respective configurations but with perfect electric conductors, are indicated as well (with the superscript $P E C$ ). A good convergence to this limit is observed for the high-frequency inductance of the copper lines.

Fig. 8 (b) shows the inductive and resistive coupling between lines 1 and 2, respectively, 3 and 4, again compared with the rectangular conductor case. The mutual resistance elements are negative, but very small with respect to the resistance elements shown in (a), such that the resistance matrix remains positive-definite. The mutual inductance elements are important, due to the close vicinity of the conductors.

\section{Micromachined Coplanar Waveguide}

The final presented example treats the coplanar waveguide (CPW) structure shown in Fig. 9. As a result of the etching process during the manufacturing of the $\mathrm{CPW}$, the silicon substrate material $\left(\epsilon_{r}=11.7\right)$ is partly removed underneath the separation between the signal line and the reference conductors. The structure was taken from [10], where it was simulated for PEC conductors. The characteristic impedance of the line for the copper conductor case $\left(\sigma_{\mathrm{Cu}}=57.2 \mathrm{MS} / \mathrm{m}\right)$ is shown Fig. 10, and at the highest frequencies approaches the PEC limit obtained from [10]. As claimed in the Introduction, this example shows that the DtN operator combined with the integral equation techniques of [6] can handle non-planar substrates including very thin conducting slabs. The dashed lines shown on the substrate in Fig. 9 denote its division in subregions for which the DtN operator is determined separately, i.e., 4 rectangles and 3 triangles. Of course, it was also necessary to determine the DtN operator for the three rectangular copper conductors.

\section{CONCLUSION}

The presented Iterative Combined Waveguide Modes algorithm leads to the Dirichlet to Neumann operator for an

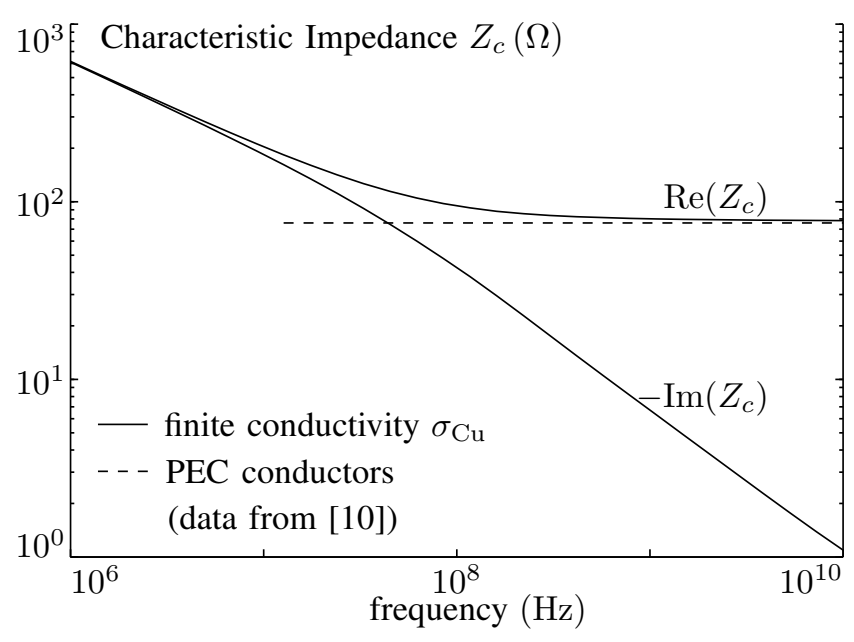

Fig. 10. The characteristic impedance $Z_{c}$ of the structure shown in Fig. 9.

arbitrary triangle, which can be used to calculate the multiconductor transmission line parameters for polygonal conductor structures. The iterative method has good convergence properties and is accurate over a broad frequency range. As an illustration, the inductive and resistive behavior of trapezoidal conductors is investigated in a few numerical examples.

\section{APPENDIX}

This Appendix displays the explicit forms of the relevant expansion matrices, in the order of their introduction in Sections II and III.

\section{A. Submatrices Related to Expanding $\psi$ over Triangle $T$}

$$
\begin{aligned}
& {\left[\mathrm{Q}_{1}\right]_{n, m}=2 \int_{0}^{1}(\sin n \pi s) t_{c_{1}, m}(s) d s} \\
& {\left[\mathrm{D}_{1}\right]_{n, n}=\left(\gamma_{n}^{-}(1)\right)^{-1} \quad \text { (diagonal) }} \\
& {\left[\mathrm{P}_{21}\right]_{n_{2}, n_{1}}=-4 j \frac{x_{0}-x_{1}}{x_{0}} \frac{\beta_{n_{1}}}{y_{0}} \frac{n_{1} \pi}{y_{0}} \frac{n_{2} \pi}{y_{0}}(-1)^{n_{1}}} \\
& \times\left(2 e^{j \beta_{n_{1}} y_{0}}(-1)^{n_{1}+n_{2}} \cos \left(\frac{n_{1} \pi x_{1}}{x_{0}}\right)-\gamma_{n_{1}}^{+}(1)\right) \\
& \times\left(\left(\frac{\pi}{y_{0}}\right)^{2}\left(n_{2}+n_{1} \frac{x_{0}-x_{1}}{x_{0}}\right)^{2}-\beta_{n_{1}}^{2}\right)^{-1} \\
& \times\left(\left(\frac{\pi}{y_{0}}\right)^{2}\left(n_{2}-n_{1} \frac{x_{0}-x_{1}}{x_{0}}\right)^{2}-\beta_{n_{1}}^{2}\right)^{-1} \\
& {\left[\mathrm{P}_{31}\right]_{n_{3}, n_{1}}=-4 j \frac{x_{1}}{x_{0}} \frac{\beta_{n_{1}}}{y_{0}} \frac{n_{1} \pi}{y_{0}} \frac{n_{3} \pi}{y_{0}}} \\
& \times\left(2 e^{j \beta_{n_{1}} y_{0}} \cos \left(\frac{n_{1} \pi x_{1}}{x_{0}}\right)-(-1)^{n_{3}} \gamma_{n_{1}}^{+}(1)\right) \\
& \times\left(\left(\frac{\pi}{y_{0}}\right)^{2}\left(n_{3}+n_{1} \frac{x_{1}}{x_{0}}\right)^{2}-\beta_{n_{1}}^{2}\right)^{-1} \\
& \times\left(\left(\frac{\pi}{y_{0}}\right)^{2}\left(n_{3}-n_{1} \frac{x_{1}}{x_{0}}\right)^{2}-\beta_{n_{1}}^{2}\right)^{-1}
\end{aligned}
$$




\section{B. Submatrices Related to $\partial \psi / \partial n$}

For the definition of $T_{11}$, the auxiliary matrices $B_{1}$ $\left(M_{1} \times M_{1}\right)$ and $\tilde{\mathrm{T}}_{11}\left(M_{1} \times N_{1}\right)$ are defined as

$$
\begin{aligned}
{\left[\mathrm{B}_{1}\right]_{\tilde{m}, m} } & =\int_{0}^{1} t_{c_{1}, \tilde{m}}(s) t_{c_{1}, m}(s) d s \\
{\left[\tilde{\mathrm{T}}_{11}\right]_{\tilde{m}, n} } & =-j \beta_{n} \gamma_{n}^{+}(1) \int_{0}^{1}(\sin n \pi s) t_{c_{1}, \tilde{m}}(s) d s
\end{aligned}
$$

and with these,

$$
\mathrm{T}_{11}=\mathrm{B}_{1}{ }^{-1} \tilde{\mathrm{T}}_{11} \text {. }
$$

With $\mathrm{B}_{2}=\mathrm{B}_{1}^{\prime}$ and $\mathrm{B}_{3}=\mathrm{B}_{1}^{\prime \prime}$ we find

$$
\begin{aligned}
& \mathrm{T}_{21}=\mathrm{B}_{2}{ }^{-1} \tilde{\mathrm{T}}_{21} \\
& \mathrm{~T}_{31}=\mathrm{B}_{3}{ }^{-1} \tilde{\mathrm{T}}_{31},
\end{aligned}
$$

with the $M_{2} \times N_{1}$ matrix $\tilde{\mathrm{T}}_{21}$ and the $M_{3} \times N_{1}$ matrix $\tilde{\mathrm{T}}_{31}$ defined as

$$
\begin{aligned}
& {\left[\tilde{\mathrm{T}}_{21}\right]_{\tilde{m}, n}=} \int_{0}^{1}\left(\frac{y_{0}}{l_{2}} \gamma_{n}^{-}(1-s) \frac{n \pi}{x_{0}} \cos \zeta_{2, n}(s)\right. \\
&\left.+\frac{x_{0}-x_{1}}{l_{2}} j \beta_{n} \gamma_{n}^{+}(1-s) \sin \zeta_{2, n}(s)\right) t_{c_{2}, \tilde{m}}(s) d s \\
& {\left[\tilde{\mathbf{T}}_{31}\right]_{\tilde{m}, n}=\int_{0}^{1}\left(-\frac{y_{0}}{l_{3}} \gamma_{n}^{-}(s) \frac{n \pi}{x_{0}} \cos \zeta_{3, n}(s)\right.} \\
&+ \\
&\left.+\frac{x_{1}}{l_{3}} j \beta_{n} \gamma_{n}^{+}(s) \sin \zeta_{3, n}(s)\right) t_{c_{3}, \tilde{m}}(s) d s
\end{aligned}
$$

\section{Submatrices Related to the Gibbs Effect Elimination}

The following functions are introduced

$$
\begin{aligned}
\hat{\gamma}^{-}(s) & \stackrel{\text { def }}{=}\left(e^{j \hat{\beta} y_{0}(1-s)}-e^{j \hat{\beta} y_{0}(1+s)}\right) \\
\hat{\gamma}^{+}(s) & \stackrel{\text { def }}{=}\left(e^{j \hat{\beta} y_{0}(1-s)}+e^{j \hat{\beta} y_{0}(1+s)}\right) \\
\hat{\zeta}_{2}(s) & \stackrel{\text { def }}{=} \frac{\pi}{2 x_{0}}\left(x_{0}+s\left(x_{1}-x_{0}\right)\right) \\
\hat{\zeta}_{3}(s) & \stackrel{\text { def }}{=} \frac{\pi}{2 x_{0}}\left((1-s) x_{1}\right) .
\end{aligned}
$$

The $M \times M$ matrices $\hat{\mathrm{D}}^{\mathrm{p}_{\mathrm{i}}}$ are found from

$$
\hat{D}^{p_{i}}=\hat{T}^{p_{i}} \hat{W}^{p_{i}}
$$

for

$$
\begin{aligned}
& \hat{\mathrm{W}}^{\mathrm{p}_{1}}=\left(\hat{\gamma}^{-}(1)\right)^{-1} \hat{\mathrm{W}}_{0}^{\mathrm{p}_{1}} \\
& \hat{\mathrm{W}}^{\mathrm{p}_{2}}=\left(\hat{\gamma}^{\prime-}(1)\right)^{-1} \hat{\mathrm{W}}_{0}^{\mathrm{p}_{2}} \\
& \hat{\mathrm{W}}^{\mathrm{p}_{3}}=\left(\hat{\gamma}^{\prime \prime-}(1)\right)^{-1} \hat{\mathrm{W}}_{0}^{\mathrm{p}_{3}} .
\end{aligned}
$$

with $\hat{\mathrm{W}}_{0}^{\mathrm{p}_{\mathrm{i}}}$ the $M$-element row vector that selects from $\Psi_{\mathrm{c}}$ a good approximation of the corner value of $\psi$ at $p_{i}$. Furthermore

$$
\hat{\mathrm{T}}^{\mathrm{p}_{1}}=\left[\begin{array}{c}
\hat{\mathrm{T}}_{11} \\
\hat{\mathrm{T}}_{21} \\
\hat{\mathrm{T}}_{31}
\end{array}\right]
$$

with

$$
\begin{aligned}
& {\left[\mathrm{B}_{1} \hat{\mathrm{T}}_{11}\right]_{\tilde{m}, 1}=-j \hat{\beta} \gamma^{+}(1) \int_{0}^{1} \cos \left(\frac{\pi s}{2}\right) t_{c_{1}, \tilde{m}}(s) d s} \\
& {\left[\mathrm{~B}_{2} \hat{\mathrm{T}}_{21}\right]_{\tilde{m}, 1}=} \\
& \quad-\frac{y_{0}}{l_{2}} \frac{\pi}{2 x_{0}} \int_{0}^{1} \hat{\gamma}^{-}(1-s) \sin \hat{\zeta}_{2}(s) t_{c_{2}, \tilde{m}}(s) d s \\
& \quad+\frac{x_{0}-x_{1}}{l_{2}} j \hat{\beta} \int_{0}^{1} \hat{\gamma}^{+}(1-s) \cos \hat{\zeta}_{2}(s) t_{c_{2}, \tilde{m}}(s) d s
\end{aligned}
$$

$$
\begin{aligned}
& {\left[\mathrm{B}_{3} \hat{\top}_{31}\right]_{\tilde{m}, 1}=} \\
& \quad \frac{y_{0}}{l_{3}} \frac{\pi}{2 x_{0}} \int_{0}^{1} \hat{\gamma}^{-}(s) \sin \hat{\zeta}_{3}(s) t_{c_{3}, \tilde{m}}(s) d s \\
& \quad+\frac{x_{1}}{l_{3}} j \hat{\beta} \int_{0}^{1} \hat{\gamma}^{+}(s) \cos \hat{\zeta}_{3}(s) t_{c_{3}, \tilde{m}}(s) d s
\end{aligned}
$$

and

$$
\hat{\mathrm{T}}^{\mathrm{p}_{2}}=\left[\begin{array}{c}
\hat{\mathrm{T}}_{31}^{\prime} \\
\hat{\mathrm{T}}_{11}^{\prime} \\
\hat{\mathrm{T}}_{21}^{\prime}
\end{array}\right] \quad \text { and } \quad \hat{\mathrm{T}}^{\mathrm{p}_{3}}=\left[\begin{array}{c}
\hat{\mathrm{T}}_{21}^{\prime \prime} \\
\hat{\mathrm{T}}_{31}^{\prime \prime} \\
\hat{\mathrm{T}}_{11}^{\prime \prime}
\end{array}\right]
$$

The $M \times M$ matrices $\hat{\mathrm{R}}^{\mathrm{p}_{\mathrm{i}}}$ are written as

$$
\hat{R}^{p_{i}}=E-\hat{L}^{p_{i}} \hat{W}^{p_{i}}
$$

with $\mathrm{E}$ the $M \times M$ unit matrix. Matrix $\hat{\mathrm{L}}^{\mathrm{p}_{\mathrm{i}}}$ is split up as

$$
\hat{\mathrm{L}}^{\mathrm{p}_{1}}=\left[\begin{array}{c}
\hat{\mathrm{L}}_{11} \\
\hat{\mathrm{L}}_{21} \\
\hat{\mathrm{L}}_{31}
\end{array}\right]
$$

with

$$
\begin{aligned}
& {\left[\mathrm{B}_{1} \hat{\mathrm{L}}_{11}\right]_{\tilde{m}, 1}=\gamma^{-}(1) \int_{0}^{1} \cos \left(\frac{\pi s}{2}\right) t_{c_{1}, \tilde{m}}(s) d s} \\
& {\left[\mathrm{~B}_{2} \hat{\mathrm{L}}_{21}\right]_{\tilde{m}, 1}=\int_{0}^{1} \hat{\gamma}^{-}(1-s) \cos \hat{\zeta}_{2}(s) t_{c_{2}, \tilde{m}}(s) d s} \\
& {\left[\mathrm{~B}_{3} \hat{\mathrm{L}}_{31}\right]_{\tilde{m}, 1}=\int_{0}^{1} \hat{\gamma}^{-}(s) \cos \hat{\zeta}_{3}(s) t_{c_{3}, \tilde{m}}(s) d s}
\end{aligned}
$$

and

$$
\hat{L}^{\mathrm{p}_{2}}=\left[\begin{array}{l}
\hat{\mathrm{L}}_{31}^{\prime} \\
\hat{\mathrm{L}}_{11}^{\prime} \\
\hat{\mathrm{L}}_{21}^{\prime}
\end{array}\right] \quad \text { and } \quad \hat{\mathrm{L}}^{\mathrm{p}_{3}}=\left[\begin{array}{c}
\hat{\mathrm{L}}_{21}^{\prime \prime} \\
\hat{\mathrm{L}}_{31}^{\prime \prime} \\
\hat{\mathrm{L}}_{11}^{\prime \prime}
\end{array}\right]
$$

\section{REFERENCES}

[1] G. Manetas, V. N. Kourkoulos, and A. C. Cangellaris, "Investigation on the frequency range of validity of electroquasistatic RC models for semiconductor substrate coupling modeling," IEEE Trans. Electromagn. Compat., vol. 49, no. 3, pp. 577-584, Aug. 2007.

[2] G. Antonini, A. Orlandi, and C. Paul, "Internal impedance of conductors of rectangular cross section," IEEE Trans. Microwave Theory Tech., vol. 47, no. 7, pp. 979-985, July 1999.

[3] K. Coperich, A. Ruehli, and A. Cangellaris, "Enhanced skin effect for partial-element equivalent-circuit (peec) models," IEEE Trans. Microwave Theory Tech., vol. 48, no. 9, pp. 1435-1442, Sept. 2000.

[4] G. Plaza, R. Marques, and F. Medina, "Quasi-TM MoL/MoM approach for computing the transmission-line parameters of lossy lines," IEEE Trans. Microwave Theory Tech., vol. 54, no. 1, pp. 198-209, Jan. 2006. 
[5] J. J. Kucera and R. J. Gutmann, "Effect of finite metallization and inhomogeneous dopings on slow-wave-mode propagation," IEEE Trans. Microwave Theory Tech., vol. 45, no. 10, pp. 1807-1810, Oct. 1997.

[6] T. Demeester and D. De Zutter, "Quasi-TM transmission line parameters of coupled lossy lines based on the Dirichlet to Neumann boundary operator," IEEE Trans. Microwave Theory Tech., vol. 56, no. 7, pp. 1649-1660, July 2008.

[7] D. De Zutter and L. Knockaert, "Skin effect modeling based on a differential surface admittance operator," IEEE Trans. Microwave Theory Tech., vol. 53, no. 8, pp. 2526-2538, Aug. 2005.

[8] T. Demeester and D. De Zutter, "Internal impedance of composite conductors with arbitrary cross section," IEEE Trans. Electromagn. Compat., vol. 51, no. 1, pp. 101-107, Feb. 2009.

[9] — "Modeling the broadband inductive and resistive behavior of composite conductors," IEEE Microwave Wireless Compon. Lett., vol. 18, no. 4, pp. 230-232, Apr. 2008

[10] H.-H. Chen, "Finite-element method coupled with method of lines for the analysis of planar or quasi-planar transmission lines," IEEE Trans. Microwave Theory Tech., vol. 51, no. 3, pp. 848-855, Mar. 2003.

[11] T. Demeester and D. De Zutter, "Applications of the Dirichlet-toNeumann boundary operator in transmission line modeling," presented at the 20th International Zurich Symposium on Electromagnetic Compatibility, Jan. 2009.

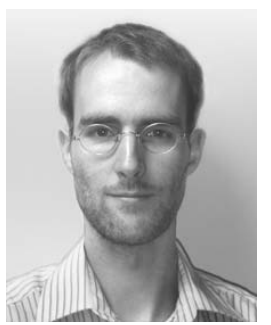

Thomas Demeester was born in 1982 in Ghent, Belgium. He received his M. Sc. Degree in electrical engineering from Ghent University in 2005, after a one-year period at ETH Zürich for his master thesis in the field of time-domain electromagnetics. $\mathrm{He}$ is currently working toward his Ph.D. degree at Ghent University, as a Research Fellow of the Fund for Scientific Research, Flanders. His research concerns electromagnetic field calculations in the presence of highly lossy media, and the development of transmission line models for interconnects.

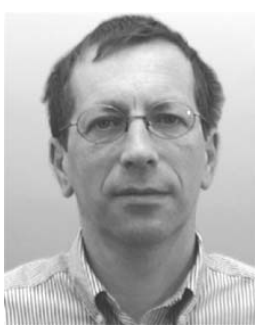

Daniël De Zutter was born in 1953. He received his M. Sc. Degree in electrical engineering from Ghent University in 1976. From 1976 to 1984 he was a research and teaching assistant at the same university. In 1981 he obtained a Ph. D. degree and in 1984 he completed a thesis leading to a degree equivalent to the French Aggrégation or the German Habilitation. From 1984 to 1996 he was with the National Fund for Scientific Research of Belgium. $\mathrm{He}$ is now a full professor of electromagnetics. Most of his earlier scientific work dealt with the electrodynamics of moving media. His research now focusses on all aspects of circuit and electromagnetic modelling of high-speed and high-frequency interconnections, packaging, on-chip interconnect and on numerical solutions of Maxwell's equations. As author or co-author he has contributed to more than 150 international journal papers. In 2000 he was elected to the grade of Fellow of the IEEE. In the past 4 yeas he served as dean of the Faculty of Engineering of Ghent University. He is an associate editor for the MTTTransactions. 